\title{
Modélisation de l'évolution à long terme de la flore adventice. II. Application à trois dicotylé- dones annuelles en un site donné
}

\author{
Philippe DEBAEKE $\left({ }^{1}\right)$ \\ I.N.R.A., Laboratoire d'Agronomie, Centre de Recherches de Grignon-Massy-Paris, F 78850 Thiverval-Grignon
}

RÉSUMÉ

\begin{abstract}
Un modèle d'évolution quantitative de la flore adventice annuelle, dont les principes ont été décrits dans un précédent travail (Debaekt \& Sebillotte. 1988), est appliqué à 3 espèces dicotylédones communes et à dynamique contrastee, issues de populations naturelles du champ d'essai de Grignon (Yvelines).

La valeur des parametres résulte du suivi de 64 parcelles, réparties sur 2 années et couvrant une diversité de systèmes de culture; cette estimation est complétée par des résultats provenant de la littérature.

La confrontation des stocks semenciers prévus et observés, après 17 annécs de culture et pour 30 parcelles labourées, permet un classement satisfaisant des parcelles testées, les écarts au modèle étant attribuables à des importations de semences, à de mauvaises estimations de productions semencières récentes et à l'hétérogénéité des stocks initiaux.

La relative concordance des taux de croissance annuels du stock simulés à Grignon et déduits des sondages sur le site de Dijon rend plus crédibles les cinétiques d'évolution du stock et confère une valeur plus générale au jeu de paramètres retenu.
\end{abstract}

Mots clés additionnels : Système de culture, désherbage. Anagallis arvensis L.. Capsellà bursa-pastoris Moench., Fallopia convolvulus (L.) Löve. weeds on a given site.

A descriptive model of the quantitative evolution of the annual weed flora, the principles of which have been exposed in earlier work (Debaeke \& StbillotTl:, 1988), has been applied to 3 broad-leaved weeds, common but with contrasting dynamics, coming from natural populations of the experimental area in Grignon (Yvelines, France). The parameter values used were derived from a survey of 64 plots, spread over 2 successive years and covering a large diversity of cropping systems, and were also taken from published results. The comparisons between predicted and observed seed banks, after 17 years of cropping on 30 ploughed plots, led to a satisfactory classification of the plots tested; differences between observed and expected values were due to some seed imports, to bad estimation of recent seed production and to the hetcrogeneity of the initial sced banks. There was fairly good agrecment in seed-bank annual growth rates simulated in Grignon and determined by sampling on an experimental field at Dijon. This confirms the value of the results on the long-term kinetics of seed banks and suggests that the empirical parameters used may be more generally applicable.

Additional key words: Cropping system, weed control. Anagallis arvensis $L$., Capsella bursa-pastoris Moench., Fallopia convolvulus $(L$.j Löve.

\section{INTRODUCTION}

Dans le précédent article de cette série (DEBAEKE \& Sebillotte, 1988), un essai de modélisation de l'évolution pluri-annuelle du stock semencier d'une parcelle, destiné à prévoir les effets à moyen et long terme des systèmes de culture sur la flore adventice a été proposé et discuté. Si sa construction est assez générale, l'application de ce modèle, dans sa forme actuelle, doit tenir

(1) Adresse actuelle: I.N.R.A., Station d'Agronomic, Centre de Recherches de Toulouse, BP 27, F 31326 Castanet-Tolosan Cedex. 
compte néanmoins d'une série d'hypothèses simplificatrices : absence de concurrence entre adventices, pas ou peu d'échanges de semences avec l'extérieur du système parcelle-couche labourée, pas d'introduction ni de disparition d'espèces sur la période de test du modèle. Ces conditions caractérisent les parcelles peu infestées (c'est-à-dire régulièrement et efficacement désherbées) et les espèces annuelles dont les semences sont persistantes et migrent peu (plantes basses, absence d'organes de dissémination). Dans ce cas, l'évolution quantitative de la flore est assez lente (BARRALIS et al., 1978).

Après paramétrage du modèle dans les conditions d'un champ d'essai, la validation est entreprise pour 3 dicotylédones annuelles fréquentes dans nos régions (BARRAlis, 1977) : Anagallis arvensis L., Capsella bursapastoris Moench., Fallopia convolvulus (L.) Löve. Celleci est un préalable nécessaire au développement prévisionnel d'un tel outil.

\section{CONDITIONS EXPÉRIMENTALES}

\section{A. Matériel d'étude}

L’analyse de la dynamique de communautés adventices naturelles, réalisée sur le dispositif expérimental de Grignon (Yvelines. France) au cours de 2 campagnes culturales (1984-85 et 1985-86). a servi de base à l"établissement du modèle et à l'estimation de ses paramètres constitutifs (DEBAEKE, 1988 $a$ et $b$ ). Les caractéristiques permanentes du site ont été décrites par HUET (1977): rappelons que le sol est un limon argileux, neutre et moyennement calcaire, non hydromorphe et peu battant. Les 2 années d'étude se distinguent notamment par la pluviométrie automnale, dont le déficit en 85-86 a déplacé la levée des adventices vers le printemps. Confrontées à la normale 1947-84, ces années ont en commun : 1) des périodes de gel intense et prolonge en janvier-février ; 2) une remontée assez lente des températures après l'hiver ; 3) une sécheresse prononcée dès le mois de mai, concentrant l'essentiel des levées en avril.

Un réseau de 64 parcelles, semées pour l'essentiel en blé d'hiver, cv. Fidel, a servi de base à la détermination expérimentale des paramètres (tabl. 1). Les valeurs du taux de levée $\alpha$, du taux de mortalité par ombrage $\delta$ et de la production de semences par plante $\mathrm{p}$ en résultent directement. Celles de $\mu$, taux de mortalité hivernale, sont simulées à partir de lois établies sur le site. Il erl va de même pour le taux de mortalité par désherbage $\beta$, dont les valeurs proviennent en partie de simulations annuelles (courbe de levée indexée au climat) et de données bibliographiques (sensibilité aux herbicides). Par contre, le taux de mortalité des semences dans le sol, $\gamma$, est uniquement déduit de la littérature.

Parmi ces situations, 30 parcelles labourćes sont retenues pour la validation. Elles different par les successions de cultures et les modes de désherbage pratiqués sur la période 1967-84, le choix de celle-ci étant lié à la disponibilité des enregistrements culturaux. Cet échantillon comprend des monocultures de céréales d'hiver et de printemps, ainsi que des rotations associant céréales et betterave, maïs ou pomme de terre. Le désherbage dépend du degré d'intensification de la culture et de la
TABLEAU 1

Répartition des 64 parcelles utilisées pour le paramétrage du modèle. selon l'année el la culture en place.

Distribution of the 64 plots used for the estimation of the model parameters, according to the year and the crop present.

\begin{tabular}{lccccccccc}
\hline Annce & Culture & BH & OH & BP & OP & MG & FH & BS \\
\hline $84-85$ & 22 & 1 & 3 & 1 & 2 & 2 & 0 \\
\hline $85-86$ & 22 & 3 & 5 & 1 & 1 & 0 & 1
\end{tabular}

BH : blé d'hiver ; OH : orge d'hiver; BP : blé de printemps; OP : orge de printemps; $\mathrm{MG}$ : mais grain; $\mathrm{FH}$ : féverole d'hiver; BS : betterave sucrière.

$\mathrm{BH}$ : winter wheat: $\mathrm{OH}$ : winter barley; $\mathrm{BP}$ : spring wheat; $\mathrm{OP}$ spring barley; $\mathrm{MG}$ : maize; $\mathrm{FH}$ : winter ficld bean ; $\mathrm{BS}$ : sugar beet

flore présente; c'est ainsi qu'une trentaine d'herbicides ont été employés de 1967 à 1984, complétés en betterave par un ou plusieurs binages mécaniques ou manuels. Sur chacune de ces parcelles, le stock semencier est estimé pour les 3 espèces.

Les 3 dicotylédones annuelles choisies sont fréquentes et abondantes à Grignon mais présentent des répartitions variables selon les systèmes de culture. Elles se distinguent par leur période de germination (MONTEGUT, 1975), la persistance de leurs semences (ROBERTS \& FEAST, 1972 ; Roberts \& BodDrell, 1983) et par les conditions de retour au sol de leurs semences (DEBAEKE \& Sebillotte, 1988).

\section{B. Méthodes d'estimation des paramètres}

- Sn: Le stock semencier parcellaire est estimé à l'automne 1984 sur la couche $0-30 \mathrm{~cm}$, à partir de 60 carottes de sol issues d'un échantillonnage systématique. Après extraction physique, on procède à une numération directe des semences, selon une procédure décrite ailleurs (DEBAEKE, 1988a), aboutissant à l'évaluation d'une densité de semences bien conformées extérieurement sur l'épaisseur maximale de la couche labourée. La précision d'échantillonnage est évaluée à laide d'une relation empirique entre variance et moyenne (BARRALIs et al., 1986; DEBAEKE, 1987), permettant de s'affranchir d'un traitement individuel systématique des carottes de sol.

- $\alpha$ : En zone non désherbée l'année de l'observation. un comptage des levées est effectué sur des cadres permanents de $0,24 \mathrm{~m}^{2}$ ( 25 à 30 par parcelle), 4 à 6 fois entre le déchaumage du précédent et la récolte de la culture. Le rapport de cette densité de plantes annuelles au stock semencier initial permet d'estimer le taux de levée $\alpha$

- $\mu$ : La comparaison des densités de plantes pré- et post-hivernales définit le taux de mortalité hivernale.

- $\delta$ : De la même manière, le taux de mortalité $\delta$ résulte de la confrontation du nombre de plantes observées à la récolte à la somme des plantes présentes en sortie d'hiver ou ayant levé au printemps.

- p : La production de semences est évaluée sur pied à la récolte de la culture par comptage direct des organes pour une espèce peu prolifique comme Fallopia convolvulus. La part de semences exportées avec la 
récolte est estimée à partir du pourcentage de plantes grimpantes. Pour les espèces à capsules (Anagallis arvensis et Capsella bursa-pastoris), on procède en 2 étapes: 1) comptage du nombre de fruits par plante ; 2) estimation sur 50 capsules du nombre de semences bien conformées par fruit. Le nombre de fleurs est également compté en vue de l'estimation de la production semencière potentielle.

\section{RÉSULTATS ET DISCUSSION}

\section{A. Estimation des paramètres}

\section{Détermination expérimentale des paramètres $\alpha, \delta, p, \varepsilon$}

\section{a) Taux de levée annuel $\alpha$}

Les valeurs moyennes du rapport flore levée/stock semencier $(\alpha)$ figurent au tableau 2, regroupées par espèce et par époque de semis de la culture sans distinction des années d'étude. Le taux de levée est maximal pour les semis de mars dans le cas des espèces à pic de levée printanier préférentiel (A. arvensis) ou absolu ( $F$. convolvulus), alors qu'on n'observe pas de différences avec les semis d'automne pour $C$. bursapastoris, espèce à germination étalée sur l'année (Roberts \& Feast, 1970 ; Montegut, 1975). Pour ces 3 espèces, de plus faibles taux sont obtenus lors de semis tardifs (maïs), les conditions hydriques s'opposant à une germination prolongée.

Il nous manque cependant des valeurs pour caractériser la levée de $F$. convolvulus en culture d'été et celle des 3 adventices en culture de betterave ou de pomme de terre (semis d'avril). Dans le premier cas, nous avons eu recours aux données d'enquête régionale (BARRALIS, 1977 ) en utilisant le rapport des fréquences de l'espèce dans les diverses cultures, conscients néanmoins que celui-ci ne dépend pas que des divers taux de levée, ce qui donne une valeur calculée de 0,065 en culture de maïs. Pour les semis d'avril, considérant une dégradation progressive des conditions de levée au cours du printemps, nous avons retenu des valeurs intermédiaires à celles des époques de semis de mars et de mai.

Une pondération arbitraire de ce taux $(\alpha / 2)$ a été adoptée pour tenir compte d'années sèches, dont les répercussions sur la levée des plantes diffèrent néanmoins selon les espèces:

- C. bursa-pastoris germe tout au long de l'année en raison d'une variabilité des états dormants et des conditions de levée de la dormance et de l'aptitude des semences non dormantes à germer dans une gamme étendue de températures (RoBERTS \& FEAST, 1970; POPAY \& ROBERTS, 1970; Dumas et al., 1976). Par ailleurs, l'imbibition rapide des téguments et la fraction importante de semences peu ou pas dormantes dans la population assure une levée précoce de cette espèce en conditions favorables (Roberts \& PotTer, 1980). C'est pourquoi, on trouve chaque année, du moins sur notre période d'étude (1967-1984), des époques où la germination n'est pas limitée (DEBAEKE, 1987); il est alors raisonnable d'admettre pour une date de semis donnée
TABLEAU 2

Valeurs expérimentales moyennes du taux de levée a pour 3 espèces adventices al 3 époques de s'mis de la culture.

Mean experimental values of the emergence rate $\alpha$ for 3 weed species and 3 crop seeding dates.

\begin{tabular}{|c|c|c|c|c|}
\hline Espèce & Epoque de semis & $\begin{array}{c}\text { Automne } \\
1\end{array}$ & $\begin{array}{c}\text { Mars } \\
2\end{array}$ & $\begin{array}{c}\mathrm{Mai} \\
3\end{array}$ \\
\hline $\begin{array}{l}\text { Anagallis } \\
\text { arvensis }\end{array}$ & $\begin{array}{l}\text { moyenne } \\
\text { ecart-lype } \\
\text { effectif }\end{array}$ & $\begin{array}{c}0,044 \\
0.0 ! 4 \\
21\end{array}$ & $\begin{array}{c}0,068 \\
0,023 \\
4\end{array}$ & $\begin{array}{c}0.016 \\
0.002 \\
3\end{array}$ \\
\hline $\begin{array}{l}\text { Capsella } \\
\text { bursa- } \\
\text { pastoris }\end{array}$ & $\begin{array}{l}\text { moyenne } \\
\text { écart-type } \\
\text { effectif }\end{array}$ & $\begin{array}{c}0.034 \\
0.018 \\
23\end{array}$ & $\begin{array}{c}0,036 \\
0,020 \\
4\end{array}$ & $\begin{array}{c}0,013 \\
0,011 \\
3\end{array}$ \\
\hline $\begin{array}{l}\text { Fallopia } \\
\text { convolvulas }\end{array}$ & $\begin{array}{l}\text { moyenne } \\
\text { écart-type } \\
\text { effectif }\end{array}$ & $\begin{array}{c}0.076 \\
0,061 \\
13\end{array}$ & $\begin{array}{c}0,092 \\
0,089 \\
4\end{array}$ & - \\
\hline
\end{tabular}

une valeur constante de $\alpha$ et un déplacement possible dans l'année des périodes de germination.

- A. arvensis germe principalement au printemps, mais également à l'automne en conditions douces (Montegut, 1975; Roberts \& Boddrell, 1983). Adoptant une démarche similaire à la précédente, il apparaît que la levée automnale est compromise 1 année sur 2. La valeur de $\alpha$ est alors réduite lorsque les conditions printanières sont également sèches, ce qui n'est le cas qu'en 1976.

- F. convolvulus germe strictement au printemps, avec un pic en avril dans nos conditions (MonTEGUT, 1975), la stratification hivernale étant nécessaire à la levée de dormance et les températures élevées induisant une dormance secondaire dès le mois de mai (RoBERTS \& FEAST, 1970 ; ROBERTS \& NEILSON, 1980). Ces exigences germinatives étroites conduisent 8 années sur 17 à des valeurs réduites de $\alpha$, par suite de printemps secs, cohérentes avec la variabilité observée (tabl. 2).

\section{b) Mortalité par ombrage $\delta$}

Les valeurs empiriques de $\delta$ varient selon le type de peuplement cultivé (vitesse de couverture du sol, port végétatif et croissance potentielle) et la nature de l'adventice (tolérance à l'ombrage et aptitude à s'affranchir du recouvrement). De manière générale, $\delta$ diminue pour les 3 espèces dans le sens céréales d'hiver, céréales de printemps, cultures à large interrang, l'orge de printemps se comportant de manière comparable au blé d'hiver (couverture rapide du sol) (tabl. 3). A. arvensis et C. bursa-pastoris ont un comportement comparable (espèces basses), alors que $F$. convolvulus, en partie par son aptitude à l'enroulement (HumE et al., 1983), est moins affectée par l'ombrage des céréales d'hiver. Nous n'avons pas introduit ici, pour une même culture, d'effet de la variété ou de l'état de croissance du peuplement, les valeurs de $\delta$ étant peu différentes pour des cultures normalement denses (DEBAEKE, 1988b). On retient ainsi pour les types distingués plus haut la valeur moyenne de $\delta$, avec un risque d'erreur supérieur pour les cultures peu couvrantes et pour une espece comme $F$. convolvulus, peu dense et à port végétatif diversifié, qui se caractérise par des taux de mortalité assez variables (tabl. 3). 
TABLEAU 3

Valeurs expérimentales movennes du taux de mortalitc par ombrage ò pour 3 espèces adventices et 4 types de peuplement cultivé. Mean experimental values of the mortaliy by shading rate for 3 weed species and 4 types of crops.

\begin{tabular}{|c|c|c|c|c|c|}
\hline Espèce & $\begin{array}{c}\text { Type de } \\
\text { peuplement }\end{array}$ & 1 & 2 & 3 & 4 \\
\hline $\begin{array}{l}\text { Anagallis } \\
\text { arvensis }\end{array}$ & $\begin{array}{l}\text { moyenne } \\
\text { écart-type } \\
\text { effectil }\end{array}$ & $\begin{array}{c}0,90 \\
0,16 \\
34\end{array}$ & $\frac{0.83}{\ldots}$ & $\begin{array}{c}0,48 \\
0,21 \\
14\end{array}$ & $\begin{array}{c}0,33 \\
0,13 \\
3\end{array}$ \\
\hline $\begin{array}{l}\text { Capsella } \\
\text { bursa- } \\
\text { pastoris }\end{array}$ & $\begin{array}{l}\text { moyenne } \\
\text { écart-type } \\
\text { effectif }\end{array}$ & $\begin{array}{c}0,90 \\
0.09 \\
39\end{array}$ & $\frac{0,82}{2}$ & $\begin{array}{c}0.67 \\
0.28 \\
13\end{array}$ & $\begin{array}{c}0,33 \\
0,13 \\
5\end{array}$ \\
\hline $\begin{array}{l}\text { Fallopia } \\
\text { convolvalus }\end{array}$ & $\begin{array}{c}\text { moyenne } \\
\text { ecart-type } \\
\text { ellectif }\end{array}$ & $\begin{array}{c}0,64 \\
0,21 \\
18\end{array}$ & - & $\begin{array}{c}0.28 \\
0.27 \\
1.3\end{array}$ & - \\
\hline
\end{tabular}

1 : blé et orge d’hiver : 2 : orge de printemps : 3 : blé de printemps ; 4 : cultures à large interrang (betterave, féverole d’hiver, maïs).

1 : winter wheat and barley $; 2:$ spring barley: $3:$ spring wheat : $4:$ crops with broad inter-row (sugar beet. winter field bean, maize).

TABLEAU 4

Valeurs expérimentales movennes de la production de fruits at de semences par plante pour 3 espèces adventices et 3 types de peuplement cultivé. Mean experimental values of fruit and seed numbers per plant for 3 weed species and 3 types of crops.

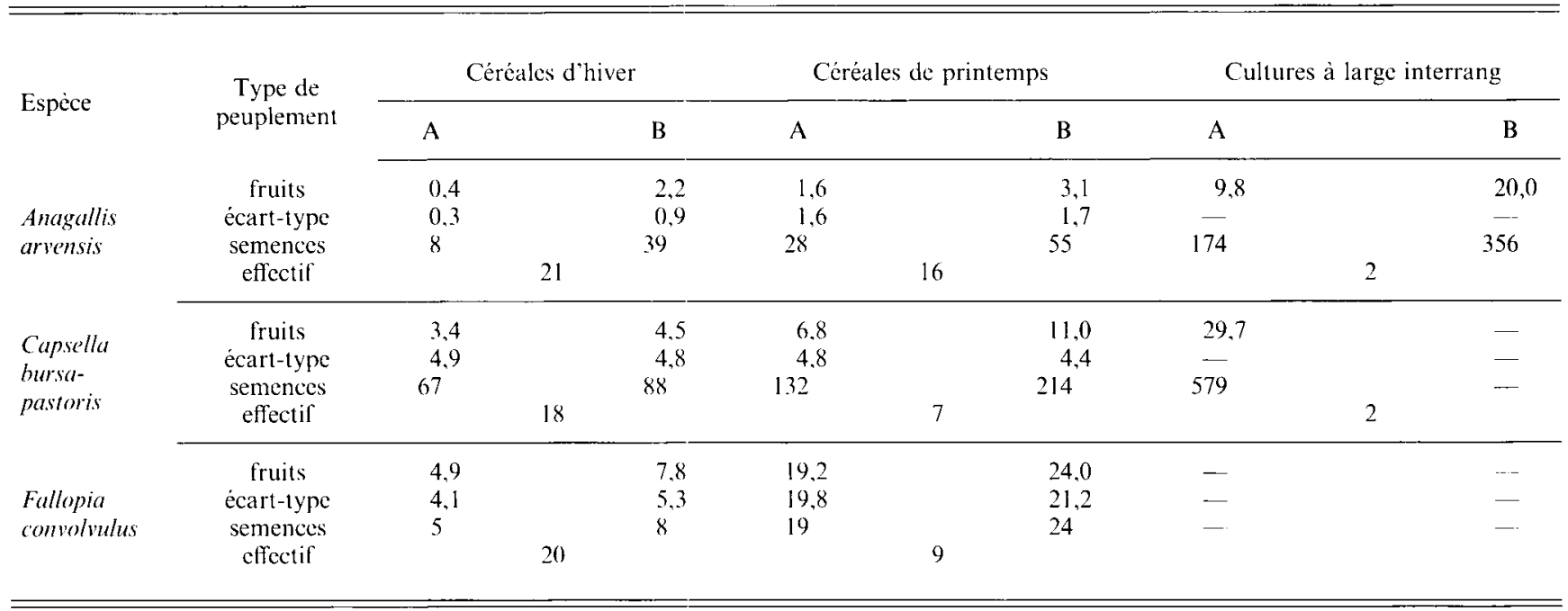

A : production réelle (issue des fruits présents à la recolte).

B : production potentielle (issue des fruits et des fleurs présentes).

A : actual production (coming from the fruits present at harvest).

B : potential production (coming from the fruits and flowers present).

\section{c) Production de semences par plante $p$}

Le tableau 4 présente par type de peuplement. les productions de fruits réelle $A$ (c'est-à-dire observées à la récolte de la culture) et potentielle $B$ (tenant compte également de la fructification complète des fleurs observées à cette époque) par plante prélevée à la récolte. A partir de capsules issues de diverses parcelles, le nombre moyen de semences bien conformées par fruit $(18 \pm 5$ pour $A$. arvensis, $20 \pm 10$ pour $C$. bursa-pastoris) est en accord avec les résultats de la littérature (SALISBURY, 1961; HURKA \& HAASE, 1982). La plasticité de la production semencière est importante, notamment pour les espèces à levée échelonnée ( $C$. bursa-pastoris en blé d'hiver, cf. tabl. 4). Les valeurs obtenues en céréales sont néanmoins faibles en regard de la littérature (SLA-
VNIC, 1962; PAWLOWSKI, 1966) et traduisent des situations de concurrence sévères imposées par la culture.

\section{d) Retour au sol et exportation des semences}

Les 3 espèces diffèrent par leurs risques d'enfouissement avant fructification et d'exportation de semences (Debaeke \& Sebillotte, 1988) :

- Par son port étalé, A. arvensis est peu affectée par les engins de récolte; une évolution des fleurs en fruits est possible, le cycle des plantes étant borné par le déchaumage, survenant à des dates variables après la récolte, du moins dans nos conditions. Aussi, la production de semences retournant au sol est jugée égale en moyenne à $\mathrm{A}+\mathrm{B} / 2$ et le taux de semences exportées $\varepsilon$ est nul. 
- Pour C. bursa-pastoris, la dissémination est quasi complète à la récolte et les fleurs observées, souvent à l'état de boutons floraux, ont peu de chances d'évoluer. Aussi, la production retournant au sol est jugée égale à $A$ et $\varepsilon$ est nul.

- Dans le cas de F. convolvulus, on estime à 40 p. 100 le taux de plantes grimpantes dont la production est exportée intégralement avec la céréale, ce qui est cohérent avec les résultats de PAwlowski \& Wszolek (1978) qui ont quantifié ces contaminations. On retiendra ainsi une valeur de $0,60(\mathrm{~A}+\mathrm{B}) / 2$ pour la production de semences restituées au stock de la parcelle.

\section{Simulation annuelle de la valeur des paramètres à partir de lois empiriques}

a) Calcul de la courbe de levée annuelle et de la valeur de $\alpha$, taux de levée dans l'interculture

La répartition dans l'année de la levée diffère entre espèces, entre années et même entre parcelles une même année (date de semis) comme l'illustrent nos résultats en culture d'hiver (tabl. 5). Ces données ont permis un ajustement aux sommes de températures efficaces (zéro de germination, humidité du sol non limitante) qui sert de base à la définition des cohortes, groupes de plantes à levée rapprochée et comportement démographique comparable.

Pour C. bursa-pastoris, la répartition hiver/printemps de la levée se calcule sur une base de $5^{\circ} \mathrm{C}$; le complément printanier et la levée en semis de printemps s'approchent de la même manière (tabl. 6). La pente de la relation, plus prononcée pour les semis de printemps, laisse penser qu'à cette époque, après avoir subi une stratification hivernale, les semences ne sont plus dormantes et répondent rapidement aux conditions climatiques favorables. Pour les semis d'automne, il faut admettre un seuil de degrés-jours, correspondant à la post-maturation et à l'imbibition de la semence lors de la réhumectation du sol à l'automne. De telles hypothèses sont en accord avec les travaux de POPAY \& ROBERTs (1970). La part des semences levant dans l'interculture peut être importante en conditions humides (cf. $1984: 17$ p. 100 de la levée annuelle), aussi, pour cette espèce, avons nous fait démarrer la relation précédente au déchaumage du précédent.

Pour $A$. arvensis, une base de $10{ }^{\circ} \mathrm{C}$ est retenue pour le calcul de la courbe de levée; la dormance prononcée de la majorité des semences et leur lente imbibition automnale renforcent l'écart des pentes entre semis d'automne et de printemps (tabl. 6). Les levées dans l'interculture sont peu importantes en moyenne (tabl. 5).

Dans le cas de $F$. convolvulus, la constance stricte en 85 et 86 des profils de levée sur un pas de temps mensuel, en dépit de conditions climatiques contrastées, nous a conduit à adopter une même courbe de levée chaque année, limitée strictement au printemps.

Ainsi pour Capsella, la valeur de $\alpha^{\prime}$ sera déduite de la somme de degrés-jours sur la période déchaumagelabour; pour Fallopia et Anagallis, on retiendra des valeurs constantes respectivement nulle et égale à 5 p. 100 du taux de levée $\alpha$.

\section{b) Mortalité hivernale $\mu$}

Pour C. bursa-pastoris, le taux de mortalité hivernale est lié au stade foliaire atteint par les plantes à l'époque du gel; par ailleurs, ce stade de développement est bien corrélé à la somme de températures en base $0{ }^{\circ} \mathrm{C}$ depuis le semis de la culture (Debaeke, 1988a). C'est par combinaison de ces 2 relations que l'on prédira chaque année la valeur de $\mu$ (fig. 1). La gamme de variation de ce taux simulé (tabl. 7) est étendue et fait apparaître un effet de la date de semis (et donc du précédent cultural). On note ainsi qu'en l'absence de tout désherbage, la mortalité des plantes levées à l'automne est déjà importante sauf pour les semis très précoces. La démarche est plus simple pour les 2 espèces printanières:

Répartition saisonnière de la levée adventice en culture d'hiver pour 2 années et 3 espèces (en \%).

Seasonal distribution of weed emergence in winter crops for 2 years and 3 weed species (in \%).

\begin{tabular}{|c|c|c|c|c|c|}
\hline Espèce + année & Période & Interculture & Automne & Mars + avril & Mai + juin \\
\hline $\begin{array}{l}\text { Anagallis } \\
\text { arvensis }\end{array}$ & $\begin{array}{c}84-85 \\
\mathrm{n}=8 \\
85-86 \\
\mathrm{n}=7\end{array}$ & $\begin{array}{c}3,5 \\
(0,3-14,9) \\
0,2 \\
(0-1,3)\end{array}$ & $\begin{array}{c}38,1 \\
(0-65,6) \\
6,1 \\
(0-23,7)\end{array}$ & $\begin{array}{c}34,6 \\
(9,2-48,9) \\
62,4 \\
(32,5-90,0)\end{array}$ & $\begin{array}{c}23,7 \\
(7,9-48,9) \\
28,3 \\
(0-69,1)\end{array}$ \\
\hline $\begin{array}{l}\text { Capsella } \\
\text { bursa- } \\
\text { pastoris }\end{array}$ & $\begin{array}{c}84-85 \\
\mathrm{n}=10 \\
85-86 \\
\mathrm{n}=8\end{array}$ & $\begin{array}{c}17,4 \\
(1,0-51,8) \\
0,5 \\
(0-2,3)\end{array}$ & $\begin{array}{c}52,5 \\
(30,2-73,3) \\
46,9 \\
(7,6-67,2)\end{array}$ & $\begin{array}{c}24,1 \\
(9,4-33,3) \\
43,4 \\
(21,8-62,3)\end{array}$ & $\begin{array}{c}5,9 \\
(0-13,3) \\
9,3 \\
(0-30,3)\end{array}$ \\
\hline $\begin{array}{l}\text { Fallopia } \\
\text { convolvulus }\end{array}$ & $\begin{array}{c}84-85 \\
\mathrm{n}=10 \\
85-86 \\
\mathrm{n}=10\end{array}$ & $\begin{array}{c}0 \\
(0) \\
0 \\
(0)\end{array}$ & $\begin{array}{c}0 \\
(0) \\
0 \\
(0)\end{array}$ & $\begin{array}{c}88,9 \\
(73,6-98,2) \\
88,3 \\
(75,1-100)\end{array}$ & $\begin{array}{c}11,1 \\
(1,9-26,4) \\
11,7 \\
(0-24,9)\end{array}$ \\
\hline
\end{tabular}




\section{TABLEAU 6}

Equations prédictives de la levée annuelle pour 2 espèces à germiration étalée: $\mathrm{A}$. arvensis et $\mathrm{C}$. bursa-pastoris.

Predictive equations for annual emergence of 2 species with staggered germination : A. arvensis and C. bursa-pastoris.

\begin{tabular}{|c|c|c|}
\hline $\begin{array}{l}\text { Epoque de semis } \\
\text { de la culture }\end{array}$ & $\begin{array}{l}\text { Anagallis } \\
\text { arvensis }\end{array}$ & $\begin{array}{c}\text { Capsella } \\
\text { bursa-pastoris }\end{array}$ \\
\hline Automne & $\begin{array}{c}y=0,64 x+5 \\
r=0,780^{* * *} \\
(16 \text { points })\end{array}$ & $\begin{array}{c}y=0,17 x-35 \\
r=0,583^{2 *} \\
(22 \text { points })\end{array}$ \\
\hline Printemps & $\begin{array}{c}y=0,99 x+25 \\
r=0,826^{* * *} \\
(15 \text { points })\end{array}$ & $\begin{array}{c}y=0,27 x+18 \\
r=0,770^{* * *} \\
(16 \text { points })\end{array}$ \\
\hline
\end{tabular}

$\mathrm{y}=$ Part de la levée annuelle réalisée à l"époque de l'observation.

$\mathrm{x}=$ Somme de températures efficaces, en base $5^{\circ} \mathrm{C}$ pour Capsella et $10^{\circ} \mathrm{C}$ pour Anagallis, depuis le semis de la culture (Anagallis) ou le déchaumage du précédent (Capsella à l'automne)

Corrélation significative à $5 \%\left(*^{*}\right)$ ou $1 \%(* * *)$.

$\mathrm{x}=$ Proportion of the annual emergence seen at the time of the observation.

$\mathrm{y}=$ Effective heat sum, on a $5^{\circ} \mathrm{C}$ basis for Capsella and a $10^{\circ} \mathrm{C}$ basis for Anagallis, since the crop seeding (Anagallis) or the stubble cultivation (Capsella in autumn).

Significant correlation at $5 \%\left(*^{*}\right)$ or $1 \%(* * *)$.

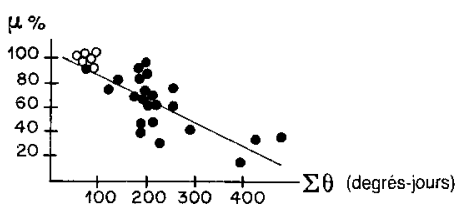

Figure 1

Relation entre le taux de mortalité hivernale $\mu$ d'une population de plantes de $\mathrm{C}$. bursa-pastoris et la somme de températures en base $0{ }^{\circ} \mathrm{C}$ sur la période semis de la culture-début du gel $(\Sigma \theta)$.

$\mu=106-0,186 \Sigma \theta r=-0,790^{* * *} n=29$ parcelles

- 1984-85 $1985-86$

Relation between the winter mortality rate $(\mu)$ of a plant population of $\mathrm{C}$. bursa-pastoris and the heat sum, on a $0^{\circ} \mathrm{C}$ basis, from seeding time to first frost $(\Sigma \theta)$.

$\mu=106-0.186 \Sigma \theta r=-0.790^{* * *} n=29$ plots

$1984-85 \bigcirc 1985-86$
- A. arvensis, au stade plantule, disparaît complètement en hiver (DEBAEKE, 1988a); on retient alors une valeur $\mu=1$, en accord avec d'autres auteurs (BARRAlis \& Chadeuf, 1980).

- F. convolvulus ne lève qu'en mars à une époque où les risques de gel intense sont quasi nuls.

\section{Paramètres issus de la bibliographie}

a) Mortalité par destruction mécanique et chimique $\beta$

Adoptant une approche décrite dans le précédent article (Debaeke \& Sebillotte, 1988) et utilisant les efficacités herbicides moyennes (pluri-annuelles et multilocales) fournies par l'ACTA (1983), on calcule pour chaque combinaison espèce-année-parcelle une valeur de $\beta$. Les références retenues pour les principales matières actives et leur variation selon le stade de l'adventice au moment du traitement sont présentées pour les céréales, à titre d'exemple, au tableau 8 . Les valeurs simulées tiennent compte également de la courbe de levée prévisionnelle évoquée précédemment; elles figurent au tableau 9, par type de peuplement, toutes parcelles et années confondues. Il apparaît ainsi que ce taux est important et peu variable pour C. bursapastoris entre céréales d'hiver et de printemps, ce qui est lié à la sensibilité des plantules à la majorité des herbicides (quel que soit leur stade) et à l'âge moyen des plantes présentes au printemps assez voisin entre peuplements. Par contre, pour les 2 espèces printanières, la différence est plus marquée entre dates de semis par suite de l'absence de désherbage de printemps certaines années ou de la précocité de ces applications peu persistantes face au pic de levée de l'espèce. La variabilité enregistrée ici est liée à celle du climat et des programmes de désherbage chimique, notamment en culture d'hiver (nouveaux produits, avancée de la date de semis).

\section{b) Mortalité in situ $\gamma$}

Ce poste est évalué à partir des résultats de la littérature en distinguant des espèces assez persistantes

TABLEAU 7

Taux de mortalité hivernale simulé pour C. bursa-pastoris : variation obtenue selon la date de semis de la culture à l'automne. Simulated winter mortality rate for $\mathrm{C}$. bursa-pastoris : variation according to crop seeding date in autumn.

\begin{tabular}{|c|c|c|c|c|}
\hline & \multicolumn{4}{|c|}{ Date de semis } \\
\hline & $<15 / 09$ & $15 / 09-14 / 10$ & $15 / 10-14 / 11$ & $\geqslant 15 / 11$ \\
\hline $\bar{X}(\%)$ & 19 & 72 & 89 & 99 \\
\hline$\sigma / \bar{X}(\%)$ & 59 & 27 & 12 & 2 \\
\hline $\mathrm{n}$ & 8 & 28 & 114 & 10 \\
\hline cultures & colza & $\begin{array}{c}\text { orge } \\
\text { blé } \\
\text { (précédent colza } \\
\text { ou céréales) }\end{array}$ & $\begin{array}{c}\text { blé } \\
\text { (tous précédents) } \\
\text { féverole }\end{array}$ & $\begin{array}{c}\text { blé } \\
\text { (précédent } \\
\text { betterave } \\
\text { ou mais) }\end{array}$ \\
\hline
\end{tabular}


TABLEAU 8

Taux de destruction moyen (en \%) des plantes adventices (présentes au moment du traitement ou sur la période d'action de I'herbicide) pour quelques produits courants en culture de céréale et variation de la sensibilité selon le stade des plantes traitées (d'après Acta, 1983).

Mean destruction rate (in \%) of weed plants (present at the time of application or during the herbicide active period in the soil) for some common mixtures in cereal crops; variation in sensitivity according to the stage of the treated plants (from Acta, 1983).

\begin{tabular}{|c|c|c|c|c|c|c|c|c|c|c|c|c|c|c|}
\hline \multirow{2}{*}{$\begin{array}{l}\text { Matières } \\
\text { actives }\end{array}$} & \multirow{2}{*}{$\begin{array}{l}\text { Dose } \\
(/ h a)\end{array}$} & \multirow{2}{*}{$\begin{array}{c}\text { Persistance } \\
\text { d'action } \\
\text { (mois) }\end{array}$} & \multicolumn{4}{|c|}{ A. arvensis } & \multicolumn{4}{|c|}{ C. bursa-pastoris } & \multicolumn{4}{|c|}{ F. convolvulus } \\
\hline & & & 1 & 2 & 3 & 4 & 1 & 2 & 3 & 4 & 1 & 2 & 3 & 4 \\
\hline Neburon & $\begin{array}{l}6 \mathrm{~kg} \\
\text { (p.c.) }\end{array}$ & 6 & 95 & 90 & 0 & 0 & 95 & 90 & 0 & 0 & 80 & 70 & 0 & 0 \\
\hline $\begin{array}{l}\text { Nitrofène } \\
+ \\
\text { linuron }\end{array}$ & $\begin{array}{c}51 \\
\text { (p.c.) }\end{array}$ & 5 & 95 & 95 & 0 & 0 & 95 & 95 & 0 & 0 & 80 & 80 & 0 & 0 \\
\hline DNOC & $\begin{array}{c}71 \\
\text { (p.c.) }\end{array}$ & 0 & 0 & 95 & 0 & 0 & 0 & 95 & 0 & 0 & 0 & 95 & 0 & 0 \\
\hline $\begin{array}{l}\text { Mecoprop } \\
\text { (sels) }\end{array}$ & $\begin{array}{c}41 \\
\text { (p.c.) }\end{array}$ & 0 & 0 & 60 & 60 & 0 & 0 & 95 & 95 & 0 & 0 & 80 & 80 & 0 \\
\hline $\begin{array}{l}\text { Bifenox } \\
+ \text { ioxynil } \\
+ \text { mecoprop }\end{array}$ & $\begin{array}{c}41 \\
\text { (p.c.) }\end{array}$ & 0,5 & 0 & 95 & 95 & 0 & 0 & 95 & 95 & 0 & 0 & 95 & 95 & 0 \\
\hline $\begin{array}{l}2,4 \mathrm{D} \\
\text { (sels) }\end{array}$ & $\begin{array}{l}0,6 \mathrm{~kg} \\
\text { (m.a.) }\end{array}$ & 0 & 0 & 80 & 80 & 70 & 0 & 95 & 95 & 90 & 0 & 80 & 80 & 70 \\
\hline
\end{tabular}

Stades : 1 : graine germée ; 2 : plantule ; 3 : plante jeune ; 4 : plante développée.

Stages : 1 : germinated seed $; 2$ : seedling ; 3 : young plant $; 4:$ developed plant.

TABLEAU 9

Valeurs moyennes par espèce du paramètre $\beta$ simulé pour 175 couples année-parcelle en céréale d'hiver et 170 en céréale de printemps.

Mean specific values of the simulated parameter $\beta$ for $175^{\prime \prime} 1$ 'ar-plot" couples of winter cereals and 170 of spring cereals.

\begin{tabular}{lcccc}
\hline \multirow{2}{*}{ Espèce } & $\begin{array}{c}\text { Période } \\
\mathrm{de} \\
\text { semis }\end{array}$ & $\begin{array}{c}\overline{\mathrm{X}} \\
(\%)\end{array}$ & Extrêmes & $\begin{array}{c}\sigma_{i} \overline{\mathrm{X}} \\
(\%)\end{array}$ \\
\hline $\begin{array}{l}\text { Anagallis } \\
\text { arvensis }\end{array}$ & automne & 51 & $5-90$ & 45 \\
\cline { 2 - 5 } $\begin{array}{l}\text { papsella } \\
\begin{array}{l}\text { bursa- } \\
\text { pastoris }\end{array}\end{array}$ & printemps & 67 & $21-99$ & 32 \\
\hline $\begin{array}{lllll}\text { Fallopia } \\
\text { convolvulus }\end{array}$ & automne & 83 & $17-100$ & 16 \\
\cline { 2 - 5 } & printemps & 86 & $32-97$ & 12 \\
\hline \hline
\end{tabular}

comme $A$. arvensis et peu persistantes comme $C$. bursapastoris et F. convolvulus (CHEPIL, 1946; ROBERTS \& FEAST, 1972 ; ROBERTS \& BODDRELL, 1983). Les taux de disparition annuels du stock, en l'absence de renouvellement, soit $\alpha+\gamma$, sont compris entre 20 et 30 p. 100 (RoBERTs \& DAwkins, 1967). Nous retiendrons un taux de mortalité in situ $\gamma$ (germination suivie de levée exclue) de 20 p. 100 pour Anagallis et de 25 p. 100 pour les
2 autres espèces, ce qui donne respectivement des taux de disparition annuels de 25 et 30 p. 100 environ.

\section{Effet des classes d'âge}

Cet effet est pris en compte implicitement dans l'estimation de $\mu$ et $\beta$. Pour p et $\varepsilon$, nous l'avons négligé car il s'est avéré peu discriminant dans nos conditions, l'âge moyen des plantes présentes à la récolte étant peu variable (DEBAEKE, 1988b). L'effet des classes d'âge sur la valeur de $\delta$ est marqué entre cohortes d'automne et de printemps (DEBAEKE, 1988b) ; or, du fait de la forte mortalité hivernale, la part des levées d'automne dans la flore présente au printemps est nulle pour $A$. arvensis et faible pour $C$. bursa-pastoris (valeur médiane de 9 p. 100), ce qui nous a conduit à ne pas considérer cet effet. La levée concentrée de $F$. convolvulus permet également de le négliger.

\section{B. Test du modèle}

\section{Principe}

La confrontation observation-prévision s'effectue à partir de l'équation suivante (DEbaeke \& SebillotTe, 1988) :

$$
\mathrm{S}_{\mathrm{n}}=\mathrm{S}_{0} \cdot \mathrm{K}_{\mathrm{n}}
$$

avec :

$\mathrm{S}_{\mathrm{n}}=$ stock semencier observé après « $\mathrm{n}$ » années d'application d'un système de culture. 
$\mathrm{S}_{0}=$ stock semencier initial non connu mais comparable entre parcelles, regroupées par unités de terrain définies par l'histoire culturale ancienne.

$\mathrm{K}_{\mathrm{n}}=$ taux de croissance cumulé du stock sur la période testée.

Le modèle sera ainsi parfaitement validé si :

- la relation $S_{n}=f\left(K_{n}\right)$ est linéaire ;

- l'ordonnée à l'origine est nulle ;

- la valeur de $S_{0}$, déduite de la régression, n'est pas aberrante.

On recherche ainsi dans un premier temps une validation globale des hypothèses et de leur assemblage sous forme de modèle.

\section{Application et discussion}

Pour les 3 dicotylédones annuelles, le test du modèle est effectué sur 30 parcelles et sur la période 1967-1984, alors que le paramétrage s'est fait en 1985 et 1986 sur ces mêmes parcelles. Les relations $S_{n}=f(K n)$ correspondantes sont présentées aux fig. $2 \mathrm{a}$, b et c. Dans chaque cas, la relation est linéaire avec un coefficient de corrélation significatif au seuil de 1 p. 100. Les écarts observation-prévision sont faibles dans l'ensemble et ne revêlent pas de biais systématique lié au groupe de parcelles ou à la succession de cultures. Cependant, pour C.bursa-pastoris, des stocks importants sont observés alors que le modèle prévoyait une forte décroissance du stock (fig. 2b). Ce groupe de 6 parcelles se caractérise soit par un sondage trop proche des allées travaillées soit par la proximité d'une prairie permanente ; en effet, dans ces zones, en l'absence de concurrence sévère, cette espèce à cycle court peut fructifier abondamment (entre 2 opérations de travail du sol ou de
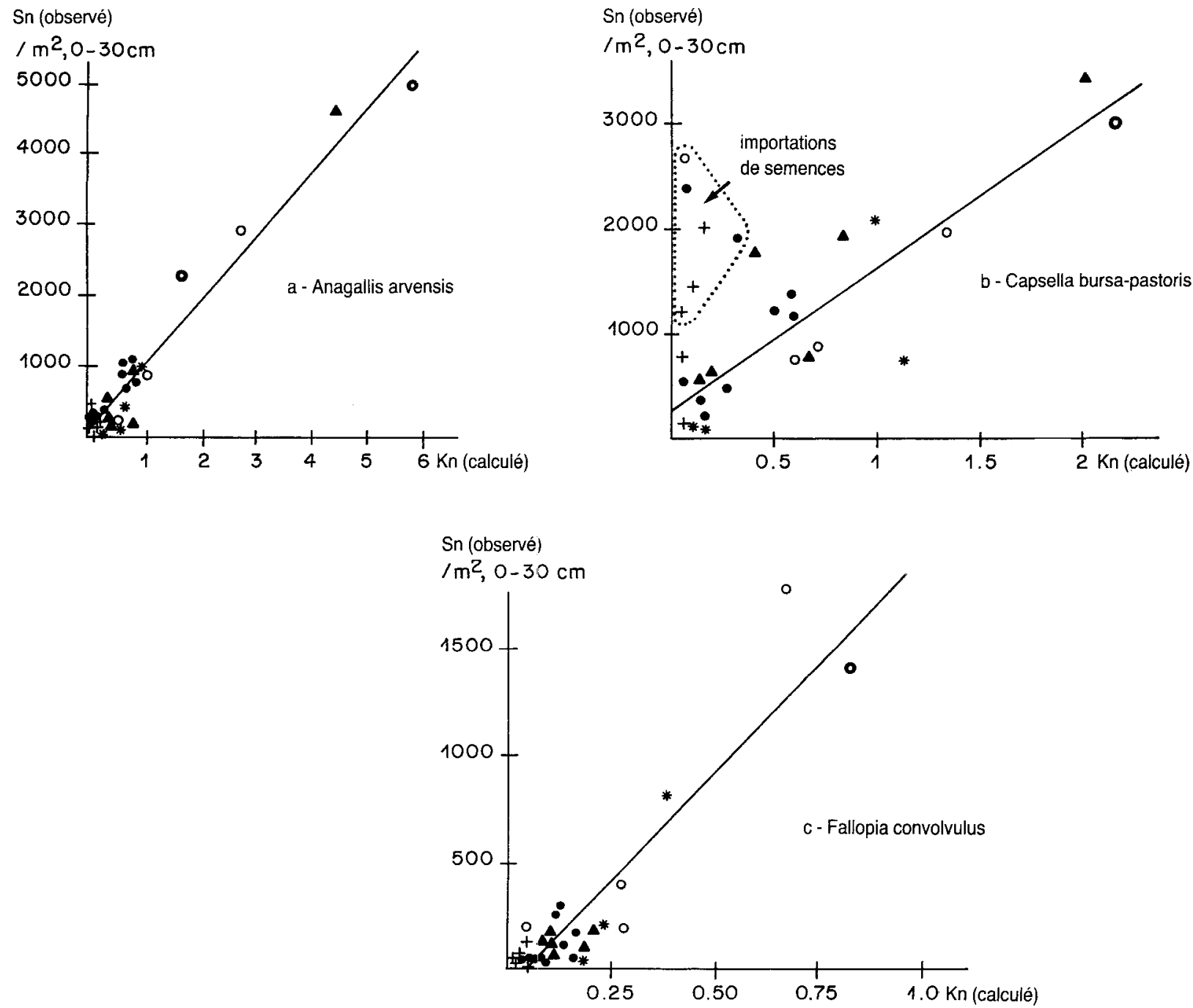

Figure 2

Test de la relation $S_{n}=f\left(K_{n}\right)$ pour 3 dicotylédones adventices.

Test of the relation $S_{n}=f\left(K_{n}\right)$ for 3 broadleaved weeds.

a. Anagallis arvensis: $S_{n}=925 K_{n}+124 \quad r=0,976^{* * *} \quad n=30$.

b. Capsella bursa-pastoris: $S_{n}=1370 K_{n}+286 \quad r=0,882^{* * *} \quad n=23$

c. Fallopia convolvulus: $S_{n}=2039 K_{n}-89 r=0,937^{* * *} \quad n=29$.

- Céréales d'hiver dominantes.

Céréales de printemps dominantes.

- Dominance of winter cereals

* Céréales + pomme de terre.

+ Céréales + maïs

Dominance of spring cereals

- Céréales + betterave.

* Cereals + potatoes.

Succession très diversifiee.

Cereals + sugar beet

- Very diversified rotation. 
fauche); les semences, nombreuses et légères sont ensuite facilement disséminées par les passages d'outil et le travail du sol. Une cartographie des levées et des stocks confirme ces hypothèses en soulignant un gradient de salissement décroissant de ces zones vers le centre des parcelles cultivées (DEBAEKE, 1987). En dehors de ces points, seuls 4 résidus suspects mais non systématiques apparaissent (2 pour Capsella, 1 pour Anagallis, 1 pour Fallopia); si l'on exclut une mauvaise évaluation du stock (échantillonnage, viabilité), il faut attribuer ces écarts à de mauvaises estimations de $\mathrm{k}_{\mathrm{i}}$, taux de croissance annuel du stock, d'autant plus marquantes sur $\mathrm{K}_{\mathrm{n}}$ qu'elles sont plus récentes; or ces parcelles se caractérisent par la présence ces dernières années de cultures peu couvrantes sur lesquelles les risques d'erreur sont supérieurs (faible nombre de situations d'étude).

L'ordonnée à l'origine n'est pas différente de la valeur nulle au seuil 5 p. 100 pour $A$. arvensis et $F$. convolvulus, et peu différente pour $C$. bursa-pastoris. L'impossibilité d'avoir $\mathrm{S}_{\mathrm{n}}=0$ pour $\mathrm{K}_{\mathrm{n}}=0$ suppose dans ce cas une contamination régulière de la parcelle, cohérente avec la discussion précédente.

La pente de la relation $S_{n}=f\left(K_{n}\right)$ traduit le stock initial moyen $\mathrm{S}_{0}$, en 1967, à une époque où le désherbage était moins systématique et moins efficace. La comparaison entre $S_{n}$ moyen et $S_{0}$ montre une stabilité du stock de $A$. arvensis $\left(\mathrm{S}_{\mathrm{n}} / \mathrm{S}_{0}=0,95\right)$ et même de $C$. bursapastoris $\left(\mathrm{S}_{\mathrm{n}} / \mathrm{S}_{0}=0,79\right)$ et une forte décroissance $\mathrm{du}$ stock de $F$. convolvulus $\left(\mathrm{S}_{\mathrm{n}} / \mathrm{S}_{0}=0,12\right)$. Les références régionales sont très partielles; GUYOT et al. (1960) concluent à un stock moyen de 700 semences $\mathrm{d}^{\prime} A$. arvensis par $\mathrm{m}^{2}$, en culture de céréales, à l'aide d'une technique (mise en germination) qui sous-estime la valeur du stock par rapport à la numération directe. Ces sens d'évolution du stock sont par ailleurs cohérents avec les cinétiques observées sur les essais de longue durée, qui suggèrent une relative stabilité d'A. arvensis et $C$. bursa-pastoris et une décroissance prononcée de $F$. convolvulus en zone régulièrement désherbée (BARRALIS \& Chadeuf, 1976; Barralis, comm. pers.).

Afin d'être plus crédible sur les étapes intermédiaires, les valeurs simulées de $\mathrm{k}_{\mathrm{i}}$, taux de croissance annuel du stock sur le site de Grignon, ont été confrontées aux valeurs observées au champ sur le dispositif de Dijon à partir des sondages annuels (BARRALIS \& CHADCEUr, 1976 ; Barralis et al., 1978 ; BARRALIS, comm. pers.). Dans l'ensemble les taux observés et simulés sont cohérents pour le blé d'hiver, l'orge de printemps et la betterave sucrière, cultures pratiquées sur les 2 sites, et ce malgré la différence d'années et de milieux (fig. 3). Cependant, on observe une plus faible gamme de variation des valeurs simulées, liée d'une part à la sousestimation probable du taux de disparition des semences in situ $(\alpha+\gamma)$ - mortalité accrue des semences d'âge inférieur à 1 an ou autres causes de mortalité au champ absentes en terrine ou en micro-parcelles - d'autre part à une surestimation des taux de mortalité de la flore levée, alors qu'on peut supposer une plus grande variabilité des conditions d'efficacité des herbicides et des états de croissance plus diversifiés du peuplement cultivé. Le taux de croissance annuel de la flore n'excède pas 5 dans nos conditions (cultures peu couvrantes), ce qui est cohérent par ailleurs avec les résultats de LEGUIZAMON \& ROBERTS (1983) en sol nu désherbé chimique- ment. Les valeurs de $\mathrm{k}_{\mathrm{i}}$ sont comparables en céréales et maïs pour les 3 dicotylédones (tabl. 10); cependant, en raison de leur prolificité supérieure, Anagallis et Capsella profitent davantage des cultures peu compétitives pour accroître leur potentiel semencier ; le niveau du stock est en effet assez lié à la fréquence de ces cultures salissantes dans la succession (cf. fig. 2).

Une analyse de sensibilité a été pratiquée sur ce modèle ; elle consiste à simuler tour à tour l'effet d'une variation de chaque paramètre - les autres paramètres restant constants - sur la valeur de $K_{n}$, taux de croissance cumulée du stock semencier. Il ressort nettement de cette analyse une forte sensibilité du modèle à de faibles variations $(+$ et $-10 \mathrm{p} .100)$ des facteurs de disparition de la flore levée ( $\delta$ et surtout $\beta$ ). Le modèle répond peu aux variations du paramètre $\alpha$, ce qui est lié à sa structure qui fait intervenir le taux de levée comme facteur de décroissance mais aussi de renouvellement potentiel du stock. Une mauvaise estimation des paramètres sensibles $(\beta, \delta, \gamma)$ pourrait être responsable des écarts enregistrés, ce que suggèrent par ailleurs les hypothèses émises pour expliquer les différences entre $\mathrm{k}_{\mathrm{i}}$ observé et simulé.

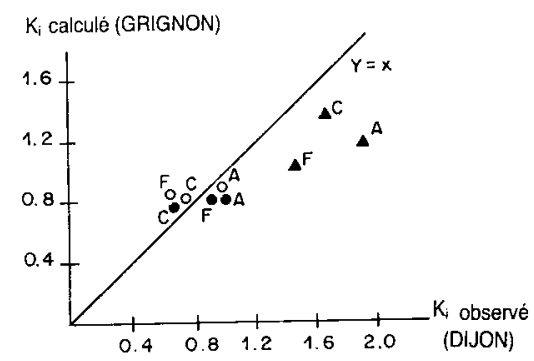

Figure 3

Comparaisom des taux de croissance anmuels du stock semencier simulés à Grignon et déduits des sondages à Dijon pour 3 adventices et 3 cultures désherbées.

- Ble dhiver Orge de printemps A Betlerave sucrière $A$ Anagallis arvensis $C$ Capsella bursa-pastoris $F$ Fallopia convolvulus

Comparison of seed bank ammual growth rates, simulated in Grignon and determined by sampling in Dijon, for 3 weed species and 3 treated crops. - winter wheat $\bigcirc$ spring barley $\Delta$ sugar beet

$A$ Anagallis arvensis $C$ Capsella bursa-pastoris $F$ Fallopia convolvulus

\section{CONCLUSION}

La confrontation réalisée montre que le modèle proposé et l'ensemble des valeurs numériques qui lui sont associées permettent de classer, de manière globalement satisfaisante, les situations culturales et les programmes de désherbage qui leur sont appliqués. Des écarts résiduels subsistent autour de la droite de régression entre valeurs observées et prévues, qui sont attribuables en partie à la variabilité initiale des stocks semenciers. Cependant, l'absence de biais systématique valide la cohérence globale des estimations. L'analyse de sensibilité suggère des voies d'amélioration possibles de ce modèle focalisées sur les 3 paramètres les plus sensibles et leurs interactions: 
TABLEAU 10

Valeurs simulées moyennes du taux de croissance ammel du stock semencier $k_{i}$ pour les principales cultures et les 3 adventices testées. Mean simulated values of the amnal growth rates of seed bank ( $\left.k_{i}\right)$ for the main crops and the 3 weeds tested.

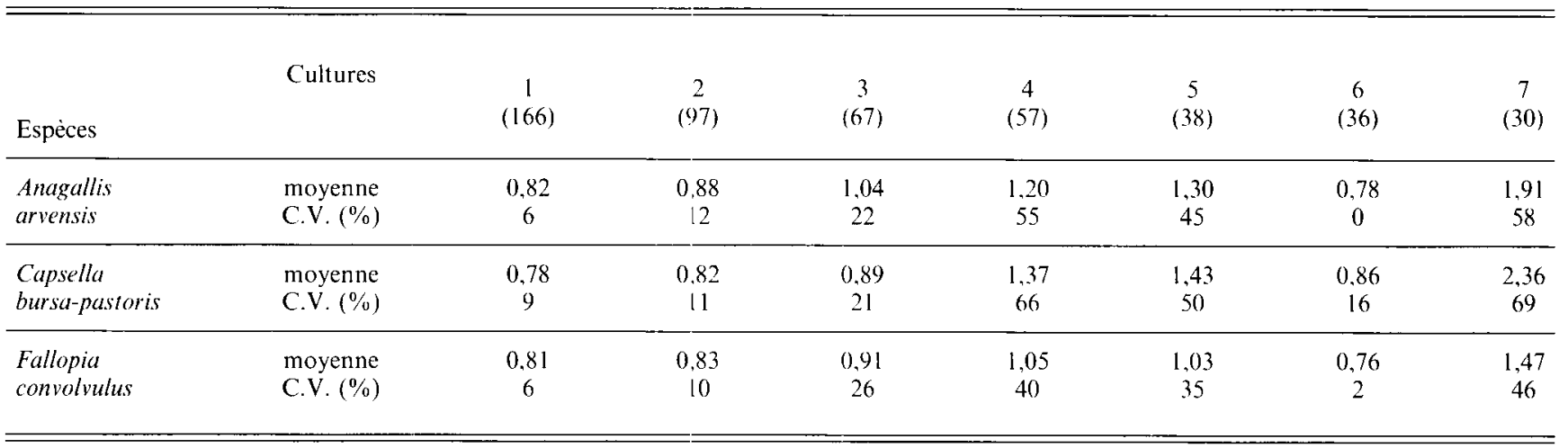

$1:$ blé + orge d'hiver $; 2:$ orge de printemps $: 3:$ blé de printemps $; 4:$ betterave sucrière $; 5:$ pomme de terre $; 6:$ maïs $; 7:$ autres cultures peu couvrantes (colza d'hiver, féverole d'hiver, lin de printemps, luzerne de $1^{\text {re }}$ année, pois de printemps).

(n) : Nombre de combinaisons culture-parcelle-année.

1: winter wheat and barley : $2:$ spring barley; $3:$ spring wheat ; $4:$ sugar beet ; $5:$ potatoes; $6:$ maize ; $7:$ other little covering crops (winter rapesced, winter field bean, spring flax, I rst year alfalfa, spring pea).

(n) : Number of combinations crop-plot-ycar.

- $\gamma$ : effet de l'âge des semences et des techniques culturales sur la mortalité in situ;

- $\beta$ : effet du climat, de l'état de surface (mottes, résidus de récolte) et du peuplement cultivé (ombrage) sur l'efficacité et la dégradation des herbicides;

- $\delta$ : effet du peuplement cultivé (croissance, structure), du climat (gel, sécheresse) et de la concurrence entre adventices sur la survie des plantules.

Ce travail a permis également de confirmer, par une approche différente, les valeurs moyennes des taux de croissance annuels du stock déterminés expérimentalement. Il reste cependant à diversifier les échelles de validation du modèle (annuelle, par comparaison avec des séries connues et intra-annuelle, par le test de la stabilité des sous-modèles) et d'en étendre l'application à d'autres sites pédo-climatiques, d'autres systèmes de culture et d'autres espèces adventices. C'est alors qu'une utilisation prévisionnelle d'un tel modèle sera envisageable pour répondre à 2 préoccupations de l'agronome :
- la prévision des répercussions à moyen et long terme sur la flore adventice des modes de conduite des cultures,

- le choix de stratégies de désherbage adaptées à des objectifs concernant le niveau ou le sens d'évolution du potentiel semencier.

Reçu le 2 décembre 1987. Accepté le 3 juin 1988.

\section{REMERCIEMENTS}

Nous tenons à remercier spécialement MM. Barralis, Perny et Sibillotte pour les lectures criticues qu'ils ont faites de ce travail. Nous sommes reconnaissants à M. BARRALIs de nous avoir communiqué des données originales obtenues sur le dispositif de Dijon. Nous ne saurions oublier $M$. Trolzifr et le personnel du Centre Expérimental de Grignon. pour le soin apporté a la réalisation du protocole.

\section{RÉFÉRENCES BIBLIOGRAPHIQUES}

ACTA, 1983. Sensibilite des mauvaises herbes aux herbicides, A.C.T.A., Paris, 211 p.

Barralis G., 1977. Répartition et densité des principales manaaises herbes en France, INRA-SFPP, $22 \mathrm{p}$.

Barralis G., Chadeuf R., 1976. Evolution qualitative et quantitative d'un peuplement adventice sous l'effet de 10 années de traitement. $5^{e}$ Col. Int. Ecol. Biol. Mauv. Herbes, Dijon. 179-186.

Barralis G., Chadeuf R., 1980. Etude de la dynamique d’une communauté adventice. I - Evolution de la flore adventice au cours du cycle végétatif d'une culture. Weed Res., 20, 231-237.

Barralis G., Chadæuf R., Compoint J. P., Gasquez J., Lonchamp J. P., 1978. Etude de quelques aspects de la dynamique d'une agrophytocénose. Proc. Symp. Mediterraneo de Herbicidas, Madrid, 85-98

Barralis G., Chadœuf G., Gouet J. P., 1986. Essai de détermination de la taille de l'échantillon pour l'étude du potentiel semencier d'un sol. Weed Res., 26, 291-297.

Chepil W. S., 1946. Germination of weed seeds. I - Longevity. periodicity of germination and viability of seeds in cultivated soil. Scient. Agric., 26, 307-346.
Debaeke P., 1987. Effets des systemes de culture sur la flore adventice dicorylédone annuelle; intérêt de la modélisation pour l'étude de l'évolution à long terme du stock de graines de lhorizon travaillé. Thèse Doct. Ing. INA-PG, 342 p. + annexes.

Debaeke, 1988a. Dynamique de quelques dicotylédones adventices en culture de céréale I - Relation flore lcvée - stock semencier. Weed Res., 28, 251-263.

Debaeke, 1988h. Dynamique de quelques dicotylédones adventices en culture de céréale II - Survie, fioraison et fructification. Weed Res., 28, 265-279.

Debaeke P., Sebillotte M., 1988. Modélisation de l'évolution à long terme de la flore adventice. I - Construction d'un modèle descriptif de l'évolution quantitative du stock de semences de l'horizon travaillé Agronomie, 8 (5), 393-403.

Dumas E., Monin J., Arnal C., 1976. Prédétermination de la dormance des graines de Capsella bursa-pastoris L. au moment de leur dissémination. $5^{e}$ Col. Int. Ecol. Biol. Mauv. Herbes, Dijon, 311-318.

Guyot L., Becker Y., Lévêque L., Barralis G., Grandjouan G., Aymonin G., 1960. Sur la présence dans les terres cultivées et incultes de semences dormantes des espèces adventices. Bull. Serv. Cart. Phytogeograph., Ser. B, CNRS, 5, 197-254 
Huet P., 1977. Contribution à l'étude des effets résiduels des cultures. Comportement du blé tendre en monoculture. Thèse Doct. Ing., Univ. Paris-Sud, 194 p. + annexes.

Hume L., Martinez J., Best K., 1983. The biology of Canadian weeds : 60. Polygonum convolvulus L., Can. J. Plan Sci., 63, 959-971.

Hurka H., Haase R., 1982. Sced ecology of Capsella bursa-pastoris L. : dispersal mechanisms and the soil seed bank. Flora, Jena, 172, 35-46.

Leguizamon E. S., Roberts H. A., 1983. Seed production by an arable weed community. Weed Res., 22, 35-39.

Montegut J., 1975. Ecologie de la germination des mauvaises herbes. In "La germination des semences». R. Chaussat \& Le Deunff Eds., Gauthier-Villars, Paris, 191-217.

Pawlowski F., 1966. Prolificacy, height and ability of producing shoots in some weed species growing among crop plants. Ann. Unir. M. Curie Sklod., Lublin-Polonia, Sect. E, 21, 175-189 (en polonais).

Pawlowski F., Wszolek M., 1978. Weediness of the summer barley and winter wheat grain on loesses and chernozems in the Hrubieszow region. Rocz. Nauk Rolnic., A. Prod. Rosl., 103, 131-145 (en polonais).

Popay A. I., Roberts E. H., 1970. Ecology of Capsella bursa-pastoris and Senecio vulgaris in relation to germination behaviour. J. Ecol., 58 , 123-139.
Roberts H. A., Boddrell J. E., 1983. Seed survival and periodicity of seedling emergence in ten species of annual weeds. Ann. appl. Biol., 102, 523-532.

Roberts H. A., Dawkins P., 1967. Effect of cultivation on the number of viable weed seeds in soil. Weed Res., 7, 290-301.

Roberts H. A., Feast P. M., 1970. Seasonal distribution of emergence in some annual weeds. Expl. Hort., 21, 36-41.

Roberts H. A., Feast P. M., 1972. Fatc of seeds of some annual weeds in different depths of cultivated and undisturbed soil. Weed Res., 12 , 316-325.

Roberts H. A., Neilson J. E., 1980. Seed survival and periodicity of seedling emergence in some species of Atriplex, Chenopodium, Polygonum and Rumex. Ann. appl. Biol., 94, 111-120.

Roberts H. A., Potter M. E., 1980. Emergence patterns of weed seedlings in relation to cultivation and rainfall. Weed Res., 20 , $377-386$.

Salisbury E. J., 1961. Weeds and Alicns. Collins, London, 384 p.

Slavnic Z., 1962. Sur la production de graines dans quelques associations de mauvaises herbes du nord de la Yougoslavie. Vegetatio. Acta Geobot., 11, 39-45. 